\title{
Emociones, Intervención Social y políticas sociales: la maternidad en la encrucijada
}

\section{Emotions, Social Intervention and social policies: motherhood at the crossroads}

\author{
ALDANA BORAGNIO \\ Instituto de Investigaciones Gino Germani (IIGG), Universidad de Buenos Aires (UBA). Centro de Investigaciones y \\ Estudios Sociológicos (CIES). ORCID: https://orcid.org/0000-0002-7082-2822.boragnio@gmail.com
}

\section{ANDREA DETTANO}

Observatorio Social, Secretaría de Ciencia y Tecnología, Universidad Nacional de La Matanza (UNLaM). Universidad de Buenos Aires (UBA). Centro de Investigaciones y Estudios Sociológicos (CIES). ORCID: https://orcid.org/0000-

0001-9985-6690. andreadettano@gmail.com

\begin{abstract}
Resumen: El presente trabajo es producto de los puntos de encuentro entre nuestras investigaciones sobre las prácticas del comer en la oficina de trabajadoras estatales y las prácticas de consumo de las destinatarias de los Programas de Transferencias Condicionadas de Ingresos, ambas con recorte geográfico en la Ciudad de Buenos Aires. Desde allí hemos identificado las emociones que se organizan en torno a la maternidad y a las prácticas de cuidado, las cuales también se vinculan a estrategias de intervención estatal que se han masificado en las sociedades latinoamericanas.

El objetivo de este escrito es recuperar el modo en que las emociones ligadas al cuidado de los hijos e hijas organizan ciertas prácticas signadas por la abnegación y sacrificialidad, donde es siempre a partir de satisfacer a otros -los/as hijos/as- que se encuentra el disfrute y la satisfacción.

Este recorrido se hizo a partir de una estrategia de indagación cualitativa, utilizando entrevistas en profundidad. Se concluye que las emociones ligadas al cuidado de los/as hijos/as vehiculizan prácticas de cuidado que colocan a las mujeres madres en la vivencia de la abnegación y la culpa.
\end{abstract}

Palabras clave: Mujeres; Prácticas de cuidado; Emociones; Maternidad; Culpa; Abnegación.

\begin{abstract}
This paper is the product of the meeting points of our investigations about the eating practices in the office and the consumption practices from the recipients of the Conditional Cash Transfers, both in the City of Buenos Aires. From these, we have identified the emotions that are organized around motherhood and care practices, which are also linked to state intervention strategies that have become widespread in Latin American societies.

The objective of this paper is to recover the way in which emotions linked to the care of sons and daughters organize certain practices marked by self-denial and sacrificiality, where it is always based on satisfying others -children- that You will find enjoyment and satisfaction. This tour will be based on a
\end{abstract}


strategy of qualitative inquiry, using in-depth interviews. It is concluded that emotions linked to the care of children convey caring practices that place women mothers in the experience of self-denial and guilt.

Keywords: Women; Emotions; Care Practices; Motherhood; feeling of Guilt; Abnegation.

\section{Introducción}

El presente escrito parte de la puesta en común de dos investigaciones: por un lado, las empleadas en oficinas de la Administración Pública Nacional (APN) en la Ciudad de Buenos Aires en relación a sus emociones y prácticas del comer (Boragnio, 2019) y, por otro, las destinatarias de Programas de Transferencias Condicionadas de Ingresos (PTCI) en la Ciudad de Buenos Aires en relación a sus prácticas de consumo (Dettano, 2019). Si bien se trata de dos investigaciones distintas, en ambos grupos las mujeres madres presentaban determinados sentires comunes en función a los modos de organizar lo atinente al cuidado de sus hijos, la reproducción de sus hogares y las formas de sentir dichas tareas. En este sentido es que este trabajo se propone describir las emociones de las mujeres trabajadoras APN y las mujeres receptoras de un $\mathrm{PTCl}$ en relación a la maternidad y al cuidado.

El diseño de ambas investigaciones fue cualitativo de tipo exploratorio descriptivo y la técnica de indagación utilizada fue la entrevista en profundidad, lo que permitió acceder a una sucesión de acontecimientos vividos por los entrevistados/as junto a la verbalización de la apropiación e interpretación que los sujetos realizan de dichos acontecimientos y los modos de sentirlos (Marradi, Archenti y Piovani, 2007). El muestreo se realizó mediante la técnica 'bola de nieve' (Taylor y Bogdan, 1987) y muestreo teórico. Entre ambos trabajos se realizaron un total de 38 entrevistas, cantidad que estuvo determinada por el criterio de saturación teórica (Glaser y Strauss, 1967).

Para el presente artículo se tomaron las entrevistas realizadas a mujeres-madres, de 19 a 56 años. Sabiendo que se trata de dos poblaciones que presentan marcadas diferencias en sus condiciones materiales de existencia, con esta puesta en común no pretendemos pasarlas por alto ni equiparar sus posibilidades, sino que buscamos poner el foco en relación a la maternidad y al cuidado de sus hijos y cómo esto supone determinados modos de sentir.

Para ello, primero realizaremos un breve recorrido por los múltiples procesos de "feminización" por los que se está atravesando; luego estableceremos nuestra perspectiva teórica sobre las emociones para reflexionar sobre la maternidad y las prácticas que habilita, centrándonos en las prácticas de cuidado; y analizaremos, a partir de material empírico, las emociones que se desprenden del lugar de mujer-madre y que las colocan en la vivencia de la abnegación y la culpa como los modos legítimos de "ser madre". Finalmente, se desarrollan algunas reflexiones finales.

\section{Algunas feminizaciones}

Las modificaciones en el mercado de trabajo, en las políticas públicas y en la organización familiar que acompañan el desarrollo del siglo XX y el comienzo del siglo XXI, produjeron cambios en la vida cotidiana de las mujeres, pero estos no solo se refirieron al trabajo extra-doméstico y a la economía, sino que su salida al mercado laboral, la extensión en el tiempo de las credenciales educativas y de formación profesional, sumada a la falta de políticas públicas en torno a los servicios de cuidado, trajo aparejados cambios en las relaciones con el ámbito doméstico y el público. Al mismo tiempo, estos cambios se sumaron a la modificación de las funciones del Estado relacionadas al cuidado y la reproducción de la vida privada. De este modo, las mujeres que no poseían capacidad económica debieron añadir a su "rol natural" de amas de casa y de cuidadoras, "algunas de las funciones cumplidas por el Estado en el pasado" (Halperin Weisburd, 2009: 51), invirtiendo una mayor cantidad de su tiempo en las tareas domésticas y comunitarias como pueden ser el manejo de los comedores barriales o la conformación de organizaciones de lucha contra la drogadicción, entre otros. Este proceso, sin embargo, no trajo aparejado un equiparamiento de los roles domésticos -aunque pudo generar una renegociación de los mismos-, ya que mayoritariamente sigue siendo la mujer la encargada y la responsable de las tareas domésticas (Rodríguez Montaño, 2008).

En Argentina, según la encuesta sobre el uso del tiempo que realizó el Instituto Nacional de Estadísticas y Censos (INDEC) en $2014,{ }^{1}$ en el promedio nacional urbano, las mujeres ocupadas utilizan 5,9 horas diarias en el trabajo doméstico no remunerado, ${ }^{2}$ mientras que, los varones ocupados utilizan un promedio de 3,5 horas. Entre los no ocupados la diferen-

1 http://www.indec.gob.ar/uploads/informesdeprensa/tnr_07_14.pdf

2 La definición de "trabajo doméstico no remunerado" utilizada se refiere a todas las actividades no remuneradas realizadas para prestar servicios para un uso final propio en el hogar. 
cia es mayor ya que las mujeres emplean 6,8 horas mientras que los varones $3,2{ }^{3}$

A estas modificaciones se le sumó el incremento de las mujeres como población económicamente activa, primero, en la producción industrial de comienzos del siglo XX como modo de bajar los costos salariales (Halperín Weisburd, 2009: 77). Y luego, con el crecimiento del trabajo administrativo, se feminizaron las ocupaciones administrativas no profesionales, profundizándose desde 1940 junto a la expansión de la administración pública (Queirolo, 2015). Actualmente, la participación de las mujeres en el empleo público dependiente del Poder Ejecutivo Nacional se mantiene en un leve y constante aumento, pasando del 28,2\% en 2014, al 33,7\% en 2016. Pero, en las instituciones de seguridad social, las mujeres ocupan el $55,5 \%$ de los puestos públicos (INDEC, 2016).

A la incorporación masiva de las mujeres como masa trabajadora se le agregan los procesos de la cada vez más intensa precarización laboral (Mészáros, 2004; Vasapollo, 2005) y la feminización de la pobreza (Fall, 2003; Tortosa, 2009), que añaden otro conjunto de desigualdades sociales asociadas con las condiciones de vida. En esta línea, el Estado ha puesto en funcionamiento diferentes intervenciones como los denominados PTCl, entendidos como transferencias de dinero a hogares en situación de pobreza y/o indigencia a cambio del cumplimiento de ciertas condicionalidades en salud, educación y/o nutrición (Cecchini y Madariaga, 2011; Rangel, 2011). Estos son, a partir de los '90, la modalidad predilecta de atención a la pobreza y alcanzaron para 2016 a 129,8 millones de destinatarios en América Latina (Cecchini y Atuesta, 2017), pero presentan un alcance mundial (De Sena, 2018) por lo que revisten una gran importancia en los modos de estructuración de lo social.

A la vez, a partir del establecimiento de los Objetivos del Milenio (ODM), ${ }^{4}$ el género ha obtenido crecientes menciones así como los objetivos de diversas políticas se han orientado a reducir las desigualdades, fomentar el empoderamiento y la igualdad entre los géneros (Bedford, 2009). Una muestra central de estas transformaciones es que en los PTCI quien asume la titularidad es la mujer-madre y, si bien, estos se encuentran generalmente destinados a los niños, son ellas las encargadas de llevar adelante toda su gestión, lo que implica un mayor trabajo para las mismas. Esto se ha sustentado y justificado aduciendo a que son las mujeres quienes se en- cargan del cuidado de los niños y del hogar, así como son ellas quienes tienen una mayor preocupación por el bienestar familiar, son más altruistas y hacen un mejor uso de los fondos. Por otra parte, se ha sostenido que el otorgamiento de estas transferencias otorgaría mayor autonomía a las mujeres (Fyszbein y Schady, 2009; Cohen y Franco, 2010).

En este sentido, las políticas sociales enfatizan el rol de la mujer en tanto "agente de cuidado", reafirmando la responsabilidad y la obligación de cuidadora y reproductora del hogar (Jelin, 2010: 76), de manera que, desde la visión estatal, el rol de la mujer está conformado a partir de su condición "natural" de madre-cuidadora (De Sena, 2014; Pautassi, 2007).

La feminización de las políticas sociales nos lleva a preguntarnos no sólo por la representación de la mujer desde el Estado, sino también cómo ésta refuerza "la distribución sexual del trabajo al interior de la familia que carga a la mujer con la <doble tarea>" (De Sena, 2014: 102) y que la mantiene en un estado de "ayudada/subvencionada". Aún más, por la masividad y alcance de las políticas en cuestión, algunos trabajos también han desarrollado el concepto de "tercera jornada" (Cena, 2017) para hacer referencia al modo en que, llevar adelante lo relacionado con la inscripción y sostenimiento de un programa social implica, para las madres titulares, un refuerzo de las desigualdades.

Como podemos observar, a pesar de los cambios innegables que se produjeron en la vida cotidiana de las mujeres, la división sexual del trabajo presenta más continuidades que rupturas.

En vista de la creciente presencia de las mujeres tanto en el empleo en la Administración Pública, como en las políticas sociales, es que intentaremos problematizar los modos en que este mayor "protagonismo" significa en aquello que todos los sujetos poseemos, que parece pertenecer al mundo privado e individual, aunque presentan un carácter eminentemente social: las emociones.

\section{Emociones, Mujeres y Maternidad}

El conocimiento del mundo se da por y a través del cuerpo y es a partir de esa relación que los sujetos experimentan la vida emocionalmente, ya que las emociones no son más -ni menos- que el resultado de las prácticas de ser, hacer y habitar el mundo. Como consecuencia de ello, estas no sólo

3 En 2015, el Ministerio Nacional de Economía realizó una encuesta sobre el uso del tiempo y los resultados fueron muy parecidos: 6,4 horas para las mujeres y 3,4 para los varones. http://www.economia.gob.ar/peconomica/basehome/DT 09 uso-del-tiempo 03.pdf

4 Entre los ocho Objetivos de Desarrollo del Milenio que propuso la Organización de las Naciones Unidas (ONU) en el año 2000 para el 2015 , el tercero es "Promover la igualdad entre los géneros y la autonomía de la mujer" y el quinto es "Mejorar la salud materna". A su vez, de los Objetivos de Desarrollo Sostenible de la ONU para el 2030, el 5 es la igualdad de género. 
tienen un carácter eminentemente social, sino que también cumplen un rol central en el desenvolvimiento de los fenómenos sociales (Bericat, 2012). De ahí que una Sociología de los cuerpos/emociones (sensu Scribano) tome como punto de partida los contextos económicos, sociales e históricos de un entramado social determinado, exhibiendo el modo en el que estos afectan y conforman los modos en los que los sujetos sienten y viven el mundo (Luna Zamora, 2005) así como la internalización de las vías disponibles de actuar y vivenciarlo.

Las relaciones sociales bajo el capitalismo requieren de un proceso de elaboración de creencias para explicar el mundo, por esto las emociones son un elemento central, ya que una indagación desde las emociones conlleva reflexionar sobre la regulación de las mismas y los modos en que las coacciones sociales externas se van convirtiendo en coacciones internas, cómo se dan las modelaciones por medio de las cuales los individuos se auto-dominan y cómo se regula la manifestación de los afectos (Elías, 2009). Desde los estudios sociales sobre cuerpos/emociones se sostiene que es a partir de unas Políticas de las emociones que se vuelven soportables "...las condiciones bajo las cuales el orden se produce y se reproduce" (Scribano, 2015a: 144) en concomitancia con una Economía Política de la Moral (Marx, [1844] 2006; Scribano, 2013) que postula en sus contenidos determinadas prácticas que devienen en imperativos morales estableciendo lineamientos en cuanto a los modos de percibir, de sentir y de actuar en el mundo (Scribano, 2015b).

En este marco, partiendo de considerar a las emociones como fenómenos sociales que se organizan y consolidan en el proceso de las experiencias históricas, nos centramos en los procesos que configuraron a la maternidad como constitutiva de emociones específicas de la mujer-madre y que se han asociado, en diferentes desarrollos y períodos, a funciones de cuidado y de renovación de los grupos sociales.

El siglo XVIII se vio atravesado por una serie de preocupaciones en torno a la elevada mortalidad infantil, lo que posó rápidamente la atención sobre los modos en que se cuidaba a los recién nacidos y se apuntó a trabajar sobre "lo doméstico" como un espacio que permitiría modificar ciertos comportamientos y prolongar la vida (Donzelot, 1998). En este sentido, el hogar se constituyó como el blanco principal sobre el cual dirigir la introducción de nuevos saberes que generaron la proliferación de una serie de tratados que contenían consejos y recomendaciones -con carácter imperativo- sobre el cuidado, a la vez que diversos documentos sobre la crianza y la educación de los niños.

La introducción de estos saberes conformó el estableci- miento de una alianza entre los médicos y las madres, a las que se les adjudicaba el lugar de ejecución de las recomendaciones y consejos (Foucault, 2000). Badinter (1981) señala el inicio de la modernidad como un período en el cual -a diferencia de la Edad Media- la función materna de cuidado investía una gran utilidad en términos de crear sujetos que compusieran la riqueza del Estado, para lo cual, los niños deberían sobrevivir y los cuidados maternales tendrían un rol de suma importancia al fortalecerse las sugerencias hacia las mujeres de ocuparse ellas mismas de sus hijos y amamantarlos. Así, las diferentes construcciones de la maternidad han estructurado unas emociones principalmente asociadas a prácticas de cuidado.

Para Dorlin, esto ha tenido lugar debido a la existencia de una "ética del cuidado". El care o cuidado debe ser entendido como "... la empatía y el sentimiento moral de solicitud que comúnmente se le adjudica a las mujeres y les sería específico" (2009: 22), lo cual, remite al sacrificio y supone la entrega de las mujeres al cuidado de otras personas. Con cuidados "... nos referimos a la gestión y a la generación de recursos para el mantenimiento cotidiano de la vida y la salud; a la provisión diaria de bienestar físico y emocional, que satisfacen las necesidades de las personas a lo largo de todo el ciclo vital. El cuidado se refiere a los bienes, servicios y actividades que permiten a las personas alimentarse, educarse, estar sanas y vivir en un hábitat propicio" (Arriagada, 2010: 58). Estos están directamente relacionadas con la preocupación, el servicio, la protección, la provisión, la contención (Schwarz, 2009), se realizan principalmente en el ámbito doméstico y requieren de "actividades emocionales, afectivas y relacionales que implican cuidados de la persona y tienen malos sustitutos en el mercado" (Carrasco, 2006: 6).

En relación a lo expuesto, articulando las emociones, la maternidad y el cuidado, nos proponemos indagar en las emociones de las mujeres entrevistadas, centrándonos en la organización de la alimentación y las prácticas de consumo, como partes constitutivas de las prácticas de cuidado llevadas adelante por las mujeres en su rol de madres.

\section{Maternidad: entre la abnegación y la culpa}

Partiendo de considerar lo hasta aquí desarrollado, en este apartado recuperamos los sentires de las mujeres-madres a partir de la realización de las variadas tareas de cuidado y reproducción del hogar, centrándonos en las prácticas ligadas al consumo, la alimentación o el cuidado de la salud y en cómo éstas son vivenciadas por las mujeres desde el sentimiento de abnegación y culpa. 
El consumo de los bienes y servicios para la reproducción de la vida cotidiana es una tarea que requiere tiempo, que es realizada principalmente por las mujeres y que se encuentra cargada de sentidos ligados a la buena administración y al conocimiento de las necesidades de cada integrante del hogar. Por su realización, las mujeres reciben como compensación cierta "virtud social" (Jelin, 2010), por ejercer roles de cuidado que "... aunque incómodo para la persona involucrada, sirve al bienestar o comodidad de, o resulta ventajoso para, los miembros más poderosos de la comunidad" (Galbraith, 1973 citado en Jelin, 2010:63).

Centrarnos en las compras rutinarias, cotidianas, relacionadas con el hogar y sus miembros son un elemento y un medio para hacer visible algo acerca de las relaciones entre las personas, así como también de sus emociones. En estas prácticas cotidianas, los consumos, pueden, no solo pensarse desde un lugar hedonista sino también como una práctica asociada muchas veces a sacrificios en el marco de relaciones afectivas, como formas de expresar amor o cuidado (Miller, 1999).

Sacrificios, bienestar ajeno y virtud social como componentes del cuidado nos condujeron a analizar la sobrecarga de tareas en las mujeres-madres, centrándonos en el concepto de abnegación, la que ha sido entendida como un "rasgo" de la buena madre (Rojas, 2007; Cutuli, 2017) a la vez que aparece como una característica central en las dinámicas familiares.

Desde los estudios sobre el cuidado, la abnegación es vista como "una capacidad o valor que permite afrontar las dificultades del cuidado y como una práctica con consecuencias positivas para quien recibe los cuidados" (Domínguez-Guedea y Díaz-Loving, 2016: 224). Si entendemos la abnegación como la acción para que los otros "sean" antes que uno (Díaz Guerrero, 1993), podemos observar que en ésta se concretan premisas socio-culturales, conformadas históricamente que mantienen a las mujeres-madres en un "siempre segundo lugar" guiando sus prácticas por sentimientos de amor y sacrificio antes que autonomía y "realización personal". En este sentido, la abnegación puede ser considerada una característica central para comprender las emociones bajo las cuales las mujeres entrevistadas construyen, asumen y mantienen la auto-responsabilización del cuidado.
¿Por qué [te traés comida] muy pocas veces?

Porque yo le preparo la vianda a la nena a la mañana y le preparo la comida a la nena para que se lleve entonces es como que me aboco a prepararle la comida a ella y no me fijo en mi comida, como sé que acá mal que mal arreglamos eso, o vamos a comer, o trae Silvia, no me preocupo mucho por mi comida.

\section{Y cuando te traés, ¿te hacés especialmente?}

¡No! No. De lo que sobró la noche anterior, olvidate. A la nena sí, todos los días me levanto a las seis de la mañana, le hago la comida fresca del día... pero para mí no, de lo que sobró de la noche anterior (40 años, trabajadora APN).

\section{¿Y vos comprás para todos, o solo para tus hijas?}

Más para ellas le compro. A veces me compro para mí (en tono muy bajo), cuando ya necesito mucho, cuando ya no tengo más, pero más para ellas. La otra que ya empieza a salir, se quiere vestir bien, le compro. (41 años, destinataria PTCI)

En cuanto a las destinatarias de los PTCl, sus emociones y prácticas de consumo, debemos considerar que los programas que se trabajaron en la investigación -Asignación Universal por Hijo y Ciudadanía Porteña- ${ }^{5}$ establecen que la titularidad en el cobro sea femenina. Es necesario poner esto en consideración ya que, en la práctica, son las mujeres quienes administran y utilizan el dinero de las transferencias. Las decisiones de consumo de las mujeres destinatarias de PTCI se ven atravesadas por los lineamientos y diseño del propio programa, ${ }^{6}$ los cuales las interpelan desde su lugar de madres, por su capacidad de administración y reconocimiento de las necesidades de los/as menores a cargo, en tanto poseedoras de determinados saberes (Villatoro, 2007; Cena, 2017).

Al centrarnos en el consumo a partir de la recepción de transferencias de ingresos, las mujeres sostienen reiteradamente pasar a un segundo plano y aplicar estrategias específicas para reducir sus gastos y "arreglárselas bastante", aunque sea "complicado".

5 Ciudadanía Porteña es un PTCI que surge en el año 2005 en la Ciudad de Buenos Aires, tiene alcance municipal y se encuentra dirigido a hogares en situación de pobreza y/o indigencia, transfiere una suma de dinero variable según la composición del hogar a través de una tarjeta magnética precargada. La Asignación Universal por Hijo para Protección Social, surge en el año 2009 y está dirigido a Niños/as y Adolescentes de hogares cuyos padres se encuentra desocupados o cuyos ingresos sean menores al Salario Mínimo, Vital y Móvil. Ambos programas concentran en la Ciudad aproximadamente 300000 destinatarios (Dettano, 2019).

6 Ciudadanía Porteña presenta la particularidad de abonarse a través de una tarjeta magnética pre-cargada, que permite comprar en los comercios adheridos al programa productos alimentarios, de higiene personal, limpieza, combustible para cocinar y artículos de librería. Esto nos permite sostener que los consumos se encuentran "condicionados". 
Crees que partir de los programas y de tener el efectivo ¿pudiste acceder a comprar más cosas?

La verdad que no porque, quizás cuando estaba sola sí, pero no recibía ningún plan... tenía la posibilidad de acceder a más cosas, pero ahora que tengo a mi hija no, es más complicado... si bien me las arreglo bastante, si cuesta mucho más, por ejemplo, si me quiero comprar algo tengo que esperar bastante hasta poder juntar, pero no... está complicado...

¿Y cómo haces si te queres comprar algo? Espero...

¿A qué? (riéndose)

Mmm...bueno en realidad desde que nació ella yo pasé a segundo plano completamente, siempre priorizo más en lo que ella necesita (...). (27 años, destinataria PTCI)

Pasa que ahora estoy en una etapa complicada con la nena, que gasta mucho en pañales y leche. Yo vivo en Lomas de Zamora, vuelvo en combi, sale cincuenta pesos, tengo el jardín que me sale seis mil pesos, estoy como complicada con la plata. La verdad es que sí, si pudiera... antes, cuando era medio soltera, comía afuera, incluso comía en Granix, ${ }^{7}$ que es caro y yo comía ahí. Pero antes no tenía el problema y ahora sí... me gusta la comida de Granix, de comer, comería eso (43 años, trabajadora APN).

Pero, es en esta resignación y sacrificio que aparece la felicidad, resultado de satisfacer a un otro/a.

¿Qué es lo que más compran?

Fideos, el aceite, yerba, mate cocido...eso es lo que más se usa. Pan, harina, para hacer unas tortas fritas, un pancito. Hay que rebuscársela. Yo soy una mina que soy muy positiva, yo no pienso...soy resolutiva. No pido ni más ni menos, lo justo y lo necesario, yo no quiero plata, no puedo tener plata, con total de sentarme y ver que mis hijos estén comiendo es la felicidad más grande que tengo, de que estén sanos, no me muevo por la plata, solo por la salud de mis hijos (52 año, destinataria PTCI).

En lo expuesto es posible observar cómo tanto las prácticas de consumo de las mujeres-madres titulares de los PTCI como los modos en que las trabajadoras de oficina organizan la alimentación del hogar, se jerarquizan a partir de un fuerte sentido de abnegación, donde "primero están ellos/as". La madre siempre resigna sus necesidades, poniéndose en segundo lugar y si, por el contrario, no actúan cumpliendo con esta jerarquización de las prácticas de consumo y de cuidado, sacrificándose, aparece la culpa, como la contracara de una abnegación que no está siendo puesta en acto.

Para Giddens la culpa es una "forma de angustia que resalta sobre todo en ciertos tipos de sociedad donde el comportamiento social está regido por preceptos morales establecidos, entre ellos los impuestos y sancionados por la tradición" (2000: 196). Estos "preceptos morales" pueden verse contenidos en las narraciones de nuestras entrevistadas, de manera que la abnegación, como virtud moral de la buena maternidad, se materializa en la sobrecarga de tareas cotidianas y en la obtención de satisfacción a través de otro, de modo que, lo que guía las decisiones de consumo son las necesidades y deseos de los hijos/as, ya que "la felicidad más grande es verlos felices a ellos".

En simultáneo, a esta presencia de la abnegación, la culpa aparece como su contracara cuando se presentan dificultades para llevar adelante las tareas de cuidado ligadas a la maternidad. Para las trabajadoras de oficina, la doble jornada de trabajo dificulta el hacerse tiempo para hacer todo lo necesario para cumplir de modo correcto con la responsabilidad moral de, en este caso, alimentar a la familia de forma apropiada.

¿Cómo te sentís con tu forma de alimentación actual? Ahora que estoy por ahí cambiando, por ahí sí. Todavía no sé. Pero no, como estaba comiendo antes no, porque soy consciente de que estaba comiendo mal y todos a mí alrededor estaban comiendo mal. También es como una carga, porque decís "si yo como mal, mis hijos comen mal y mi marido come mal", entonces si yo como bien, o sea, todos comemos bien. Pero yo tengo que ser la propulsora de esto, yo... Así que no, estaba comiendo re mal y sabía que estaba comiendo mal. Y ahora bueno, vamos a ver qué sale (22 años, trabajadora APN).

La culpa aparece ante la responsabilidad de ser quienes llevan adelante el bienestar del grupo doméstico y por no lograr que esto sea como "debe ser". Así, a partir de los preceptos morales de la "buena madre" la culpa se imprime en la cotidianeidad de la vida familiar y la auto-responsabilización del cuidado aparece como una carga que se ubica en la relación abnegación-culpa. De este modo, como nos dijo una entrevistada, ellas hacen y se auto-responsabilizan por todos los integrantes de la familia nuclear.

7 "Granix" es un restaurant vegetariano del microcentro de la Ciudad de Buenos Aires que vende comida por peso. 
Y estoy en este proceso de cambio, o sea realmente venía, estábamos medios desbarrancados digamos y nos sentimos mejor, te digo porque fue a nivel inquietud familiar digamos, si bien yo lo detecto, yo lo analizo y yo saco el turno, ya lo vimos los beneficios todos (36 años, trabajadora APN).

Las mujeres, como culpables y responsables, toman el rol de mujer-madre y asumen múltiples tareas, dejándose de lado a sí mismas, poniendo a los hijos/as como escalafón jerárquico (Roche Cárcel, 2012), quedando relegadas a lo que sobra, al picoteo o a la nada. De este modo se fortalece en la cotidianeidad el estereotipo -socialmente valorado- de mujeres-madres que dejan de lado sus deseos entregándose al cuidado de los otros. Así, la culpa aparece cuando no se satisface primero a los hijos, cuando piensan en ellas mismas, en tomarse un tiempo para alguna actividad, darse un gusto o comprarse algo que necesitan.

Y digamos, ¿con esa plata entonces comprás yogur, comprás comida?

Sí, lo único que puedo comprar más que todo porque si lo ocupo en otra cosa yo ya me siento mal, es como si le estuviera robando a ellos.

¿Pero otra cosa sería que por ejemplo?

Ah, no, como por decir dale que yo voy y le agarro y me compro una zapatilla para mí y ellos me dicen "mami con la asignación no nos has comprado nada" (31 años, destinataria PTCI).

¿Por qué notas que te cuesta incorporar ejercicio?

Porque nunca encuentro horario, o sea, tiene que ser de dos a tres de la mañana si mis hijos duermen (risas), si concretan su sueño. Me cuesta más, me cuesta más, yo creo que, porque tengo amigas que les encanta correr y llueva, truene, ellas salen a correr por más que el nene te agarre de la pierna y te diga: "Mama no te vayas", se va a correr. Se ve que no tengo esa pasión, mi marido también se va a nadar llueva, truene, me esté muriendo yo en casa, aguantame una hora que vuelvo (risas), será que no tengo esa conducta (36 años, trabajadora APN).

Como podemos observar, la culpa, ejerce un papel auto-regulador, ya que sentir culpa las devuelve a la abnegación, sea porque los niños/as reclaman, "ellas se sienten mal" o no tienen la conducta necesaria. En este sentido, como presentamos en el siguiente esquema, culpa y abnegación se presentan como un binomio estructurante del sentir de las mujeres-madres.

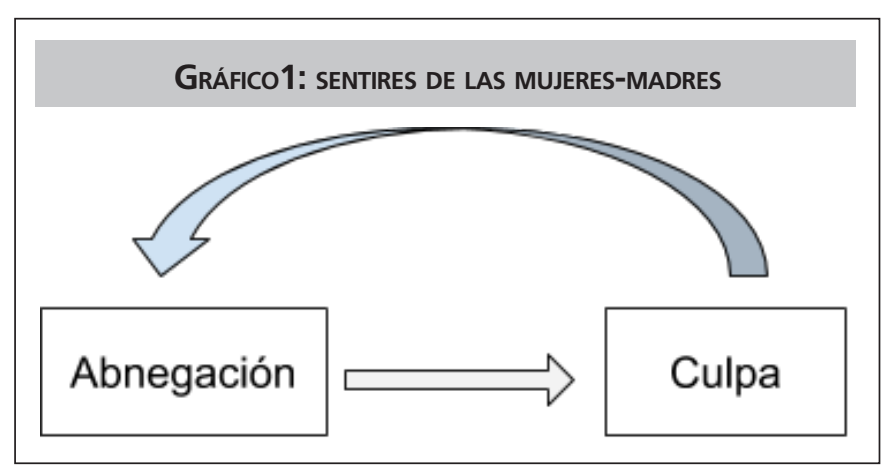

Fuente: elaboración propia

La abnegación genera el rechazo de los deseos propios y la culpa frena la vuelta de éstos como posibilidad, regresando a la abnegación como modo de ser madre que las acerca a la felicidad a partir la concreción de los deseos de los otros. Como señala una entrevistada, no es solo que no pueden ponerse en primer lugar, si no que principalmente, "no da gastar en mi".

¿Consumos para vos, como mujer, tenés alguno?

No, porque no me da gastar en mi porque necesito primero para mis hijos, a parte el tema de que están en secundaria, en la pre adolescencia, es como que en la misma escuela juega un poquito mucho eso de porque eres boliviano eres boliviana, costurero, entonces empiezan a insultar entre los chicos entonces tienen que ir si o si bien vestidos, jaja (40 años, destinataria PTCl).

\section{Reflexiones finales: La encrucijada del amor}

En estas páginas hemos considerado aquellas emociones y prácticas que se han asociado históricamente a la maternidad, así como los modos correctos de cuidar y proteger para alargar la vida. Así, en el análisis realizado fue posible observar cómo el lugar de la mujer-madre sigue apareciendo "cargado" de responsabilidades, de tareas y de emociones que las colocan en el lugar de constante sacrificio en favor de otros. De esta forma, los lugares emocionales que pueden ser habitados por estas mujeres surgen desde la abnegación, donde la vivencia de la felicidad se da a partir de satisfacer a otro, auto-colocarse en segundo lugar Y el dúo abnegación-culpa puede visualizarse a partir de considerar cómo las mujeres-madres entrevistadas organizan sus cotidianidades, su tiempo para el trabajo dentro y fuera del hogar a partir del cuidado de sus hijos/as. A la vez, sus prácticas de consumo también se organizan en torno a satisfacer todo aquello que los niños/as puedan querer, necesitar y/o reclamar. 
Así, lo que podemos sostener a partir del análisis de las entrevistas es la operatoria de una economía política de la moral conformando unos modos correctos de pensar, sentir y cuidar cuando se es madre. De esta manera, la encrucijada de la maternidad plantea cuestionar estos lugares posibles de ser habitados por las mujeres y el modo en que el lugar de madre es un ámbito cargado de regulaciones, obligaciones, moralidades, ideales de comportamiento, que se traduce en tareas de cuidado que no solo suponen la inversión de tiempo sino también unos modos de sentir específicos que reproducen y aumentan las desventajas que las mujeres acumulan en diversos ámbitos.

Asimismo, cabe la pregunta acerca de cuáles son las consecuencias del mayor protagonismo de las mujeres en los procesos de feminización e intervención estatal aludidos que se constituyen hace algunas décadas, ¿Son una buena noticia o simplemente responden a los requerimientos del régimen de acumulación e implican el sostenimiento de las desigualdades en la división sexual del trabajo y la reafirmación de unos modos de sentir que refuerzan el esquema madre-amor-cuidado-abnegación-culpa?

\section{Bibliografía}

Arriagada, I. (2010). La crisis de cuidado en Chile. Revista de Ciencias Sociales, 27, diciembre, 2010, 58-67. Universidad de la República, Uruguay.

Badinter, E. (1981). ¿Existe el amor maternal? Historia del amor maternal. Siglos XVII al XX. España: Ediciones Paidós Ibérica.

Bedford, K. (2009). Developing Partnerships. Gender, sexuality and the reformed World Bank. Minneapolis-London: University of Minnesota Press.

Bericat, E. (2012). Emociones. Sociopedia.isa, 1-13.

Boragnio, A. (2019). Comer en la oficina: prácticas del comer y emociones de mujeres trabajadoras en el ámbito de la administración pública nacional argentina. Tesis doctoral no publicada, Universidad de Alicante, Alicante, España.

Carrasco, C. (2006). La paradoja del cuidado: necesario pero invisible. Revista de Economía Crítica. N5, 39-64.

Cecchini, S. y Madariaga A. (2011). Programas de Transferencias Condicionadas. Balance de la experiencia reciente en América Latina y el Caribe. Santiago de Chile: Naciones Unidas.

Cecchini, S. y Atuesta, B. (2017). Programas de transferencias condicionadas en América Latina y el Caribe. Tendencias de cobertura e inversión. Serie: Políticas Sociales No 224. Santiago de Chile: Naciones Unidas/CEPAL.
Cena, R. (2017). Discusiones en torno a los cuidados sociales: ¿hacia una triple jornada? Reflexiones desde poblaciones destinatarias de políticas sociales., VI Encuentro Internacional sobre Vida Cotidiana, Conflicto y Estructura Social. Centro de Investigaciones y Estudios Sociológicos. Montevideo, Uruguay.

Cohen, E. y Franco, R. (2010). Programas de transferencias condicionadas: ¿pidiendo peras al olmo? Persona y Sociedad. Vol. XXIV, No 3, 91-121.

Cutuli, R. D. (2017). Género y trabajo emocional: Los fundamentos de la precariedad en el nivel inicial. Mar del Plata, Provincia de Buenos Aires. Trabajo y sociedad, (28), 37-54. Recuperado en 29 de marzo de 2019, de http:// www.scielo.org.ar/scielo.php?script=sci arttext\&pi$\mathrm{d}=\mathrm{S} 1514-68712017000100003 \& \operatorname{lng}=\mathrm{es} \& \mathrm{t} \operatorname{lng}=\mathrm{es}$

De Sena, A. (2014). Las mujeres iprotagonistas de los programas sociales? Brever aportes a la discusión sobre la feminización de las políticas sociales. En De Sena, A. (Ed.) Las politicas hechas cuerpo y lo social devenido emoción. Lecturas sociológicas de las políticas sociales. Buenos Aires: Estudios Sociológicos Sociales.

(2018). La Intervención Social en el inicio del Siglo XXI: Transferencias Condicionadas en el Orden Global. Buenos Aires: ESEditora.

Dettano, A. (2017). Prácticas de consumo y emociones de las destinatarias de programas de transferencias condicionadas de ingreso en la Ciudad de Buenos Aires. Revista Novos Rumos Sociológicos, Vol. 5, № 8, 8-32.

(2019). Políticas Sociales y Consumo: una lectura desde las emociones. Tesis doctoral no publicada, Universidad de Alicante, Alicante, España.

Díaz-Guerrero, R. (1993). Un factor cardinal en la personalidad de los mexicanos. Revista de Psicología Social y Personalidad, 9(2), 1-19.

Domínguez-Guedea, M. y Díaz-Loving, Ro. (2016). Escala de abnegación en cuidadores familiares de adultos mayores. Anales de psicología, 2016, vol. 32, n¹ (enero), 224233. http://dx.doi.org/10.6018/analesps.32.1.190411

Donzelot, J. (1998). La policía de las familias. Buenos Aires: Nueva Visión.

Dorlin, E. (2009). Sexo, género y sexualidades. Introducción a la teoría feminista. Buenos Aires: Nueva Visión.

Elias, N. (2009) El proceso de la civilización. México: Fondo de Cultura Económica.

Fall, Y. (2003). Género y pobreza. En De Villota, Paloma (ed.) Economía y género. Macroeconomía, política fiscal y liberación. Análisis de su impacto sobre las mujeres. Barcelona: Icaria editorial. 
Foucault, M. (2000). Los Anormales. Buenos Aires: Fondo de Cultura Económica.

Fyszbein, A. y Schady, N. (2009). Panorama general. Transferencias Monetarias Condicionadas. Reduciendo la Pobreza Actual y Futura. Banco Internacional de Reconstrucción y Fomento / Banco Mundial, Washington, DC.

Giddens, A. (2000). Modernidad e identidad del yo. Barcelona: Península.

Glaser, B.; Strauss, A. (1967). The discovery of grounded theory: strategies for qualitative research. New York: Aldine Publishing Company.

Halperín Weisbud, L. (2009). Cuestiones de género, mercado laboral y políticas sociales en América Latina: caso Argentina. Documentos de trabajo $N^{\circ} 13$. Buenos Aires: CEPED-UBA. Disponible en: http://biblioteca.clacso.edu. ar/Argentina/ceped-uba/20120118105631/DT13-Halperin et al.pdf

INDEC (2016). Anuario Estadístico de la República Argentina 2015 https://www.indec.gob.ar/nivel4 default.asp?id tema 1=3\&id tema $2=10$ \&id tema $3=102$

Jelin, E. (2010). Pan y afectos. La transformación de las familias. Fondo de Cultura Económica, Buenos Aires.

Luna Zamora, R. (2005). Sociología del miedo. Un estudio sobre las ánimas, diablos y elementos naturales. México: Universidad de Guadalajara.

Marradi, A.; Archenti, N. Y Piovani, J. I. (2007). Metodología de las ciencias sociales. Buenos Aires: Emece Editores.

Marx, K. [1844] (2006). Manuscritos Económico-filosóficos de 1844. Buenos Aires: Colihue.

Mészáros, I. 2004. Unemployment and Casualisation: A Great Challenge to the Left", mimeo, 2004, pp. 4/8.

Miller, D. (1999). Ir de compras. Una teoría. Madrid: Siglo Veintiuno Editores.

Pautassi, L. (2007). El cuidado como cuestión social desde un enfoque de derechos. Documento de trabajo. Serie Mujer y desarrollo. Santiago de Chile: CEPAL

Queirolo, G. A. (2015). Dactilógrafas y secretarias perfectas: el proceso de feminización de los empleos administrativos (Buenos Aires, 1910-1950). Historia Crítica, N57, Julio septiembre, 117-137.

Rangel B. M. (2011). Pobreza Rural y los Programas de Transferencias Condicionadas en América Latina y El Caribe. Documento de Trabajo $N^{\circ}$ 3. Proyecto Conocimiento y Cambio en Pobreza Rural y Desarrollo. Santiago de Chile: Rimisp.

Roche Cárcel, J.A. (2012). Entre la felicidad y la igualdad. El papel del sentimiento cultural del amor en la situación de desigualdad de la mujer española. En Bericat Alustey,
E. (Ed.) Sociologías en tiempos de transformación social. España: CIS

Rodríguez Montaño, M. F. (2008). Estado actual de las investigaciones sobre mujer casada, profesional y madre: del trabajo remunerado al trabajo doméstico. Un estudio sociológico de la familia. Revista Científica Guillermo de Ockham, 6 (2).

Rojas, M. (2007). Asociación entre la habilidad de cuidado del cuidador, el tiempo de cuidado y el grado de dependencia del adulto mayor que vive situación de enfermedad crónica, en la cuidad de Girardot. Avances de en-fermería, 25(1), 33-45. Recuperado de http://www.enfermeria. unal.edu.co/revista/

Scribano, A. (2013). Cuerpos y emociones en el capital. NÓMADAS, (39), octubre 2013, 29-45.

(2015a). Una aproximación al estado de las sensibilidades en Argentina desde la(s) política(s) de la perversión. En: Sánchez Aguirre, Rafael (Comp.) Sentidos y sensibilidades: exploraciones sociológicas sobre cuerpos/emociones. Buenos Aires: Estudios Sociológicos Editora. (pp. 141-162). Disponible en: http://estudiosociologicos.org/ portal/sentidos-y-sensibilidades-exploraciones-sociologicas-sobre-cuerposemociones/

(2015b). iDisfrútalo! Una aproximación a la economía política de la moral desde el consumo. Buenos Aires: elaleph.com

Schwarz, P. K. N. (2009). La maternidad en las prácticas y la subjetividad de las mujeres jóvenes hetero y homosexuales de clase media urbana. Un estudio cuali cuantitativo en la Ciudad Autónoma de Buenos Aires. X Jornadas Argentinas de Estudios de Población. Asociación de Estudios de Población de la Argentina, San Fernando del Valle de Catamarca.

Taylor, S. J., \& Bogdan, R. (1987). Introducción a los métodos cualitativos de investigación. Barcelona: Editorial Paidós.

Tortosa, J. M. (2009). Feminización de la pobreza y perspectiva de género. Revista Internacional de Organizaciones (RIO). N³, Diciembre 2009, 71-89. Disponible en: http:// revista-rio.org/index.php/revista rio/article/view/33/36

Vasapollo, L. (2005). Le Ragioni di una Sfida in Atto. Lavoro Contro Capitale (Precarietà, Sfruttasmento, Delocalizzazione), Jaca Book, Milão, 2005.

Villatoro, P. (2007). Las transferencias condicionadas en América Latina: luces y sombras. CEPAL: SEMINARIO INTERNACIONAL: Evolución y desafíos de los programas de transferencias condicionadas. Disponible en: https://www.cepal.org/sites/default/files/events/files/2007-nov-cepal pablovillatoro ptc.pdf 